\title{
Plant host and sugar alcohol induced exopolysaccharide biosynthesis in the Burkholderia cepacia complex
}

\author{
S. Josefin Bartholdson, ${ }^{1,2}$ Alan R. Brown, ${ }^{2}$ Ben R. Mewburn, ${ }^{3}$ \\ David J. Clarke, ${ }^{1}$ Stephen C. Fry, ${ }^{3}$ Dominic J. Campopiano ${ }^{1}$ \\ and John R. W. Govan ${ }^{2}$ \\ ${ }^{1}$ School of Chemistry, University of Edinburgh, Edinburgh EH9 3JJ, UK \\ ${ }^{2}$ Centre for Infectious Diseases, University of Edinburgh, Edinburgh EH16 4SB, UK \\ ${ }^{3}$ Institute of Molecular Plant Sciences, University of Edinburgh, Edinburgh EH9 3JH, UK
}

Correspondence

John R. W. Govan

john.r.w.govan@ed.ac.uk

Received 6 August 2007

Revised 2 May 2008

Accepted 5 May 2008
The species that presently constitute the Burkholderia cepacia complex (Bcc) have multiple roles; they include soil and water saprophytes, bioremediators, and plant, animal and human pathogens. Since the first description of pathogenicity in the Bcc was based on sour skin rot of onion bulbs, this study returned to this plant host to investigate the onion-associated phenotype of the Bcc. Many Bcc isolates, which were previously considered to be non-mucoid, produced copious amounts of exopolysaccharide (EPS) when onion tissue was provided as the sole nutrient. EPS production was not species-specific, was observed in isolates from both clinical and environmental sources, and did not correlate with the ability to cause maceration of onion tissue. Chemical analysis suggested that the onion components responsible for EPS induction were primarily the carbohydrates sucrose, fructose and fructans. Additional sugars were investigated, and all alcohol sugars tested were able to induce EPS production, in particular mannitol and glucitol. To investigate the molecular basis for EPS biosynthesis, we focused on the highly conserved bce gene cluster thought to be involved in cepacian biosynthesis. We demonstrated induction of the bce gene cluster by mannitol, and found a clear correlation between the inability of representatives of the Burkholderia cenocepacia ET12 lineage to produce EPS and the presence of an $11 \mathrm{bp}$ deletion within the bce $B$ gene, which encodes a glycosyltransferase. Insertional inactivation of bceB in Burkholderia ambifaria AMMD results in loss of EPS production on sugar alcohol media. These novel and surprising insights into EPS biosynthesis highlight the metabolic potential of the Bcc and show that a potential virulence factor may not be detected by routine laboratory culture. Our results also highlight a potential hazard in the use of inhaled mannitol as an osmolyte to improve mucociliary clearance in individuals with cystic fibrosis.

\section{INTRODUCTION}

The genus Burkholderia includes three closely related microbial species that highlight diverse evolutionary adaptation to different niches and hosts. Burkholderia mallei is a soliped-specific pathogen that only occasionally infects humans. Burkholderia pseudomallei is a free-living soil microbe and the causative agent of the subtropical human disease melioidosis. The species that presently

Abbreviations: Bcc, Burkholderia cepacia complex; CF, cystic fibrosis; CGD, chronic granulomatous disease; EPS, exopolysaccharide; HPAE$\mathrm{PAD}$, high-performance anion-exchange chromatography with pulsed amperometric detection; TFA, trifluoroacetic acid.

Three supplementary tables and a supplementary figure are available with the online version of this paper. constitute the Burkholderia cepacia complex (Bcc) have multiple roles; they include soil and water saprophytes, rhizosphere parasites, bioremediators, plant growth promoters and plant, animal and human pathogens. Members of the Bcc are particularly associated with life-threatening respiratory infections in patients with chronic granulomatous disease (CGD), and are the most potentially virulent, transmissible and inherently resistant microbes to have emerged as cystic fibrosis (CF) pathogens in recent decades (Govan, 2006; Mahenthiralingam et al., 2005).

Although most species within the Bcc produce a variety of putative virulence factors, the role of these factors in the pathogenesis of human infection is unclear (Mahenthiralingam et al., 2005). Evidence from various model systems (mouse, rat, plant and nematode) suggests 
that the importance of individual virulence factors, or combinations of factors, depends on the infection model used (Bernier et al., 2003). In addition, studies of Bcc infections in CF patients also suggest a key role of hostpathogen interactions, since clinical outcome in individual patients cannot be predicted even during epidemic outbreaks when multiple patients are infected by the same strain (Govan et al., 1993). The first description of pathogenicity in the Bcc was based on sour skin rot of onion bulbs (Burkholder, 1950). In this study, we returned to this plant host to investigate the onion-associated phenotype of the Bcc, and reveal a link between growth conditions and exopolysaccharide (EPS) production.

EPS is a putative Bcc virulence factor that is involved in persistence of the bacteria in CF lungs (Conway et al., 2004), interactions with antimicrobial peptides (Herasimenka et al., 2005) and the formation of biofilms (Cunha et al., 2004). The EPSs of Burkholderia species have recently been comprehensively reviewed (Goldberg, 2007). Recent studies (Zlosnik et al., 2008) have also challenged the previous belief that mucoid, EPS-producing colonial morphotypes of $\mathrm{Bcc}$ are rare in both environmental and clinical isolates (Govan \& Deretic, 1996). Other studies have shown that mucoid Bcc isolates mostly synthesize one type of EPS, with a highly branched heptasaccharide repeating unit, which was named cepacian (Moreira et al., 2003; Sist et al., 2003). EPS production has been shown to increase when the Bcc are grown in mannitol-rich yeast extract medium (MYEM) (Sage et al., 1990; Zlosnik et al., 2008).

Here we report the novel observation that many Bcc isolates, found to be non-mucoid on typical culture media, produce copious amounts of EPS when onion tissue is provided as the sole nutrient. Chemical and molecular analyses suggest that EPS biosynthesis is strain-specific and that the plant compounds responsible are primarily sugars and sugar alcohols. We show that the EPS phenotype on onion media is associated with the previously described bce cluster (Moreira et al., 2003), thought to be involved in cepacian biosynthesis.

\section{METHODS}

Bacterial strains and culture conditions. Bcc isolates used in this study are described in Table 1 and include 16 isolates from the two published Bcc strain panels (Coenye et al., 2003; Mahenthiralingam et al., 2000). Additional Bcc strains investigated included Burkholderia pyrrocinia BTS7, Burkholderia cenocepacia BTS2, as well as 19 Burkholderia multivorans, 14 Burkholderia cenocepacia IIIA and 11 Burkholderia cenocepacia IIIB isolates from our collection. Isolates were recovered from storage at $-80{ }^{\circ} \mathrm{C}$ by subculture on nutrient agar (NA; Columbia base agar, Oxoid) and subsequently grown on media composed of $1.5 \%(\mathrm{w} / \mathrm{v})$ bacteriological agar (Oxoid) containing $2 \%(\mathrm{w} / \mathrm{v})$ sugars and fractions from various isolation methods below.

Sugars and other related chemicals were purchased from SigmaAldrich, BDH AnalaR, MP Biochemicals, Fluka BioChemika or Acros Organics.
Onion maceration. White onion slices were placed in Petri dishes and inoculated with stationary-phase Bcc cultures $\left(10^{6}\right.$ c.f.u. $)$ that had been cultured overnight in $2.5 \%(\mathrm{w} / \mathrm{v})$ nutrient broth No 2 (Oxoid) with $0.5 \%(\mathrm{w} / \mathrm{v})$ yeast extract. The onions were left at $30{ }^{\circ} \mathrm{C}$ for 5 days. The results were assessed by eye, and onion maceration recorded as positive or negative.

Onion extract agar. Peeled white onions (1 kg) were chopped, homogenized in a blender at room temperature, and filtered through muslin. The filtrate was filter-sterilized through a $0.22 \mu \mathrm{m}$ filter and lyophilized to give a yellow sticky powder (typical yield $62 \mathrm{~g}$ ). Twenty grams of lyophilized onion extract and $15 \mathrm{~g}$ bacteriological agar were made up to 11 with distilled $\mathrm{H}_{2} \mathrm{O}$, then autoclaved at $121{ }^{\circ} \mathrm{C}$ for $15 \mathrm{~min}$. Strains were subcultured onto onion agar and incubated for $72 \mathrm{~h}$ at $30{ }^{\circ} \mathrm{C}$. Mucoidy was recorded on a scale ranging from nonmucoid $(-)$ to very mucoid $(+++)$.

Sugar agar. Sugar agar contained $20 \mathrm{~g}$ of the sugar of interest, $2 \mathrm{~g}$ yeast extract, and $15 \mathrm{~g}$ bacteriological agar dissolved in 11 distilled $\mathrm{H}_{2} \mathrm{O}$ (Sage et al., 1990). The sugars used were as follows: D-fructose, D-galactose, D-mannitol, D-glucose, glycerol, lactose, L-rhamnose, Dmannose, maltose, sucrose, myo-inositol, ribitol (adonitol) and Dglucitol (sorbitol). The fructan polysaccharide inulin was also tested.

As a control, isolates were grown on bacteriological agar containing $0.2 \%(\mathrm{w} / \mathrm{v})$ yeast extract alone.

Reverse-phase chromatography. Twenty millilitres of onion extract, $2 \%(\mathrm{w} / \mathrm{v})$ in distilled $\mathrm{H}_{2} \mathrm{O}$, was loaded onto a pre-packed C8 column (10 g/60.0 ml, Varian, Anachem) and bound material was eluted stepwise using three concentrations of methanol (20\%, $50 \%$ and $80 \%, v / v$; Fisher Scientific). Each fraction was lyophilized, dissolved in distilled $\mathrm{H}_{2} \mathrm{O}$ and incorporated into $1.5 \%(\mathrm{w} / \mathrm{v})$ bacteriological agar.

Ethyl acetate partitioning. To separate any lipids, non-polar, nonacidic and polar compounds, the resolubilized onion extract was brought to $\mathrm{pH} 7.0$ using $\mathrm{NaOH}$ and partitioned against ethyl acetate (Fisher Scientific) at $1: 1(\mathrm{v} / \mathrm{v})$. The phases were separated, and the aqueous phase was brought to $\mathrm{pH} 2.0$ using $\mathrm{HCl}$ and the extraction was repeated. Following both extractions, the organic and aqueous layers were evaporated or lyophilized respectively, and then redissolved in distilled $\mathrm{H}_{2} \mathrm{O}, \mathrm{pH}$ adjusted to 7.0, and incorporated into $1.5 \%(\mathrm{w} / \mathrm{v})$ bacteriological agar.

Acid hydrolysis. The aqueous phase residue of the ethyl acetate partitioned onion extract was redissolved in distilled $\mathrm{H}_{2} \mathrm{O}(50 \mathrm{mg}$ $\mathrm{ml}^{-1}$ ) and was hydrolysed in $2 \mathrm{M}$ TFA (trifluoroacetic acid; Sigma) at $60{ }^{\circ} \mathrm{C}$ or $120{ }^{\circ} \mathrm{C}$ for $1 \mathrm{~h}$.

Paper electrophoresis. To fractionate the extract based on the presence or absence of functional groups, the freeze-dried aqueous phase of the onion extract was weighed and resuspended in distilled $\mathrm{H}_{2} \mathrm{O}$ to a final concentration of $50 \mathrm{mg} \mathrm{ml}^{-1}$. One millilitre was loaded at a central origin on a Whatman no.1 paper $(57 \times 42 \mathrm{~cm})$. The following standards were added in the margins of the paper: glucose 6-phosphate, glucose, glucosamine, methyl green (Sigma) and orange $\mathrm{G}(\mathrm{BDH})$. Electrophoresis was conducted at $1 \mathrm{kV}$ for $20 \mathrm{~min}$, in volatile buffers at $\mathrm{pH} 2.0$ or 6.5 with white spirit or toluene as coolant (Fry, 2000).

Paper chromatography. One millilitre aliquots of each of the redissolved aqueous phase of the onion extract and the TFAhydrolysed samples $\left(50 \mathrm{mg} \mathrm{ml} \mathrm{m}^{-1}\right.$ ) were chromatographed on Whatman no. 1 paper alongside markers (ferulic acid, rhamnose, glucose, lactose, mannose, galactose, fructose, mannitol and glycerol) in butan-1-ol/acetic acid/water (12:3:5, by vol.) for up to $60 \mathrm{~h}$. 
Table 1. EPS biosynthesis of $B$. cepacia complex species when grown on agar supplemented with various substrates

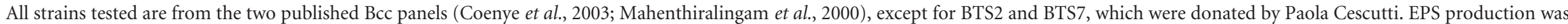
scored on a scale from - (no EPS) to +++ (very mucoid). Mucoid growth described as +++ is shown in Fig. 1(b). YE, yeast extract agar.

\begin{tabular}{|c|c|c|c|c|c|c|c|c|c|c|c|c|c|c|}
\hline Species & Strain & Source & YE & Onion & Glucose & Sucrose & Fructose & Inulin & Glycerol & Mannitol & Glucitol & Ribitol & Inositol & Mannose \\
\hline B. cepacia & ATCC 25416 & Onion & - & ++ & - & ++ & +++ & - & +++ & +++ & +++ & +++ & +++ & + \\
\hline B. cepacia & CEP509 & $\mathrm{CF}$ & - & - & - & + & ++ & + & ++ & ++ & + & + & + & - \\
\hline B. multivorans & C1576 & $\mathrm{CF}$ & - & ++ & - & + & +++ & + & ++ & +++ & +++ & +++ & +++ & - \\
\hline B. multivorans & ATCC 17616 & Soil & - & ++ & - & + & +++ & + & ++ & +++ & +++ & +++ & +++ & - \\
\hline B. cenocepacia & $\mathrm{J} 2315^{\star}$ & $\mathrm{CF}$ & - & - & - & - & - & - & - & - & - & - & - & - \\
\hline B. cenocepacia & $\mathrm{K} 56-2^{*}$ & $\mathrm{CF}$ & - & - & - & - & - & - & - & - & - & - & - & - \\
\hline B. cenocepacia & $\mathrm{BC} 7 *$ & $\mathrm{CF}$ & - & - & - & - & - & - & - & - & - & - & - & - \\
\hline B. cenocepacia & PC184 & $\mathrm{CF}$ & - & ++ & - & + & ++ & + & + & ++ & + & + & + & - \\
\hline B. cenocepacia & BTS2 & $\mathrm{CF}$ & - & ++ & - & + & ++ & + & + & ++ & ++ & + & ++ & - \\
\hline B. stabilis & LMG14294 & $\mathrm{CF}$ & - & - & - & - & + & - & - & + & - & - & - & - \\
\hline B. vietnamiensis & LMG10929 & Rice & - & +++ & - & ++ & +++ & + & +++ & +++ & + & - & +++ & ++ \\
\hline B. vietnamiensis & PC259 & $\mathrm{CF}$ & - & ++ & - & + & +++ & + & ++ & +++ & + & - & - & - \\
\hline B. dolosa & E12 & $\mathrm{CF}$ & - & ++ & - & - & +++ & - & ++ & ++ & - & + & +++ & - \\
\hline B. ambifaria & AMMD & Soil & - & +++ & - & + & +++ & + & +++ & +++ & +++ & +++ & +++ & - \\
\hline B. anthina & $\mathrm{W} 92^{\mathrm{T}}$ & Soil & - & +++ & - & - & +++ & + & +++ & +++ & +++ & +++ & +++ & ++ \\
\hline B. anthina & $\mathrm{C} 1765$ & $\mathrm{CF}$ & - & + & - & - & +++ & - & +++ & +++ & - & +++ & + & + \\
\hline B. pyrrocinia & BTS7 & $\mathrm{CF}$ & - & +++ & - & + & +++ & +++ & +++ & +++ & +++ & +++ & +++ & + \\
\hline B. pyrrocinia & C1469 & $\mathrm{CF}$ & - & ++ & - & ++ & ++ & + & ++ & +++ & +++ & +++ & ++ & - \\
\hline
\end{tabular}

${ }^{\star}$ ET12 isolates. 
Staining and elution methods. Electrophoretograms and chromatograms were stained with silver nitrate (Fry, 2000) to reveal monosaccharides, oligosaccharides, alditols, saccharinic acids and phenols, and with aniline hydrogen phthalate to reveal monosaccharides and reducing disaccharides. The paper strips of interest from both methods were eluted by a syringe method with distilled $\mathrm{H}_{2} \mathrm{O}$ (Eshdat \& Mirelman, 1972). The eluted material was incorporated into $1.5 \%(\mathrm{w} / \mathrm{v})$ bacteriological agar with $0.2 \%(\mathrm{w} / \mathrm{v})$ yeast extract.

High-performance anion-exchange chromatography with pulsed amperometric detection (HPAE-PAD). Twenty microlitres each of the aqueous phases of the onion extract and the TFAhydrolysed samples $\left(0.1 \mathrm{mg} \mathrm{ml}^{-1}\right)$ were analysed by HPAE-PAD (Dionex). The system consisted of an AS3500 autosampler, GP40 gradient pump, ED40 electrochemical detector, and PC10 pneumatic controller. The amperometry detector cell contained a gold electrode and a $\mathrm{pH}-\mathrm{Ag} / \mathrm{AgCl}$ combination reference electrode. CarboPac MA-1, PA-1 and PA-100 columns and guard columns were used for the separation of alditols, monosaccharides and oligosaccharides, respectively. Eluents, degassed by bubbling with helium, were as follows:

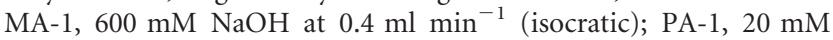
$\mathrm{NaOH}$ for $3 \mathrm{~min}$, then $\mathrm{H}_{2} \mathrm{O}$ for $32 \mathrm{~min}$, then a $0-200 \mathrm{mM} \mathrm{NaOH}$

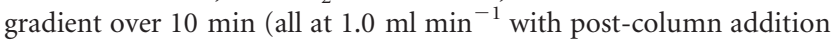
of base); PA-100, $100 \mathrm{mM} \mathrm{NaOH}$ throughout, supplemented with a 0-200 mM NaOAc gradient over $30 \mathrm{~min}$, then 200-800 mM NaOAc

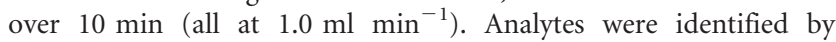
comparison of retention times to those of standards and quantified by integration of peak area with Chromeleon software (Dionex).

Investigation of conserved EPS gene clusters in Bcc species. Genome sequences representing five Bcc species were examined to determine if two previously published EPS gene clusters within $B$. cenocepacia J2315, the bce gene cluster (Moreira et al., 2003) and the wcb gene cluster (Parsons et al., 2003), are conserved across the Bcc. The amino acid sequences for every ORF within each of the two gene clusters within B. cenocepacia J2315 (genome sequence available at the Wellcome Trust Sanger Institute; http://www.sanger. ac.uk/) were used to search by TBLASTN the following Bcc genome sequences: Burkholderia ambifaria AMMD, Burkholderia vietnamiensis G4, B. multivorans ATCC 17616, Burkholderia sp. 383 (all sequences are available at the US Department of Energy Joint Genome Institute; http://www.jgi.doe.gov/) and Burkholderia dolosa AU0158 (available at the Broad Institute; http://www.broad.mit. edu/). TBLASTN searches were performed using default parameters (BLOSUM 62, Word size 3).

RT-PCR analysis of EPS biosynthetic gene clusters. The EPSproducing strain, B. ambifaria AMMD, was cultured in $0.2 \%(\mathrm{w} / \mathrm{v})$ yeast extract with or without supplementation with $2 \%(\mathrm{w} / \mathrm{v})$ mannitol. RNA was extracted from mid-exponential phase cultures (RNeasy Protect Bacteria Mini kit; Qiagen) and DNase I-treated (RNase-free DNase set; Qiagen), prior to reverse transcription with $1.5 \mu \mathrm{g}$ RNA template, random primers and SuperScript III Reverse Transcriptase (Invitrogen). cDNAs and corresponding non-RT controls were used as template in PCR reactions specific for two distinct wza homologues (Bamb_5549 and Bamb_3621) located within two separate putative EPS biosynthetic gene clusters. Primer sequences are available upon request.

PCR analysis of bceB gene. Using the $b c e B$ gene sequence of $B$. cenocepacia J2315, bceB homologues were identified within the publicly available genome sequences of $B$. ambifaria AMMD, $B$. cepacia sp. 383, B. cenocepacia AU1054, B. cenocepacia HI2424, B. cenocepacia PC184, B. vietnamiensis G4 and B. dolosa AU0158. The eight gene sequences were aligned, and PCR primers flanking the location of the previously described $11 \mathrm{bp}$ deletion in B. cenocepacia J2315 (Moreira et al., 2003) were designed based on conserved regions across the eight aligned sequences (For 5'-TGAAGGCGGT[G/ C]GCGATCGTC-3'; Rev 5'-TCGAT[G/C]CGCACGTCGTCGAG-3'). For preparation of genomic DNA, one or two bacterial colonies were resuspended in $20 \mu \mathrm{l}$ lysis solution [0.25\% (w/v) SDS, $0.05 \mathrm{M} \mathrm{NaOH}]$ and incubated at $95{ }^{\circ} \mathrm{C}$ for $15 \mathrm{~min}$. After brief centrifugation, $180 \mu \mathrm{l}$ sterile water was added and centrifugation performed at $13000 \mathrm{~g}$ for 5 min. Two microlitres of supernatant containing genomic DNA was used as template in PCR assays. PCRs were performed in a $50 \mu \mathrm{l}$ volume containing $300 \mathrm{nM}$ forward and reverse primer, $1.5 \mathrm{mM}$ $\mathrm{MgCl}_{2}, 260 \mu \mathrm{M}$ of each dNTP, $4 \%$ (v/v) DMSO, $1 \mathrm{U}$ Taq polymerase (Invitrogen) and appropriate manufacturer's reaction buffer. Thermal cycling was performed on a GeneAmp PCR System 9700 (Applied Biosystems) with the following parameters: $94{ }^{\circ} \mathrm{C} 3 \mathrm{~min} ; 40$ cycles of $94{ }^{\circ} \mathrm{C}(30 \mathrm{~s}), 60{ }^{\circ} \mathrm{C}(30 \mathrm{~s})$ and $72{ }^{\circ} \mathrm{C}(30 \mathrm{~s}) ; 72{ }^{\circ} \mathrm{C} 10 \mathrm{~min}$. PCR products were electrophoresed on a $4 \% \mathrm{E}$-Gel (Invitrogen) and visualized by UV illumination. The B. cenocepacia J2315 bceB gene sequence harbouring the $11 \mathrm{bp}$ deletion yields a PCR product of $140 \mathrm{bp}$, compared to $151 \mathrm{bp}$ from the wild-type sequence found in $B$. cenocepacia IST 432 (Videira et al., 2005).

PCR analysis of BCESM and $\mathbf{C b I A}$. Genomic DNA was prepared as described above. PCRs were performed as described previously (Mahenthiralingam et al., 1997; Sajjan et al., 1995).

Insertional inactivation of bceB in $B$. ambifaria AMMD. Insertional inactivation of $b c e B$ was performed using the pGP $\Omega T p$ suicide vector, essentially as described previously (Flannagan et al., 2007). In brief, a $300 \mathrm{bp}$ fragment internal to the bceB ORF of $B$. ambifaria AMMD and flanked by XbaI and EcoRI sites was PCRamplified and ligated into the corresponding sites in $\mathrm{pGP} \Omega \mathrm{Tp}$ following appropriate restriction. Resulting plasmids were transformed into Escherichia coli GT115 competent cells (InvivoGen) and subsequently introduced into $B$. ambifaria AMMD by triparental mating. Resulting exconjugants were selected using gentamicin $\left(50 \mathrm{mg} \mathrm{l}^{-1}\right)$ and trimethoprim $\left(100 \mathrm{mg} \mathrm{l}^{-1}\right)$, and mutants identified by PCR using a chromosomal-specific primer in conjunction with the vector-specific primer RSF1300 (Flannagan et al., 2007).

\section{RESULTS}

\section{EPS production on onion agar}

When a lyophilized onion extract was incorporated into agar at $2 \%(\mathrm{w} / \mathrm{v})$ as the sole nutrient, members of the Bcc were not only able to grow, but a majority of isolates also produced copious amounts of EPS, as typified by $B$. ambifaria AMMD (Fig. 1a, b). This phenotype is not observed when the Bcc are cultured on B. cepacia media (Mast Diagnostics), nutrient agar and other common laboratory media. Induction of EPS biosynthesis on onion agar was observed in all Bcc species investigated, but was not observed in all strains within a species (Table 1 and Supplementary Tables S1, S2 and S3, available with the online version of this paper). The exception was the single strain of Burkholderia stabilis available for testing. Of particular interest was the failure of the well-characterized B. cenocepacia ET12 representatives J2315, K56-2 and BC7 to produce EPS on onion agar. In addition, there was no correlation between induction of Bcc EPS on onion agar and the ability of individual Bcc strains to cause maceration of onion bulbs (data not shown). 
(a)

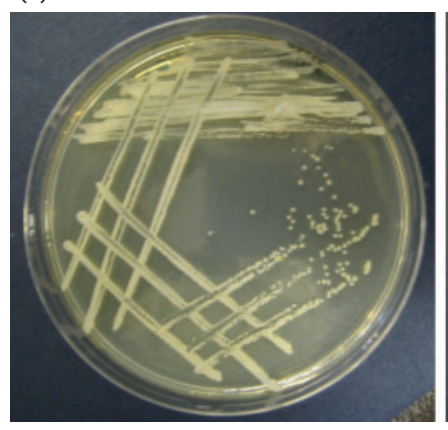

(b)

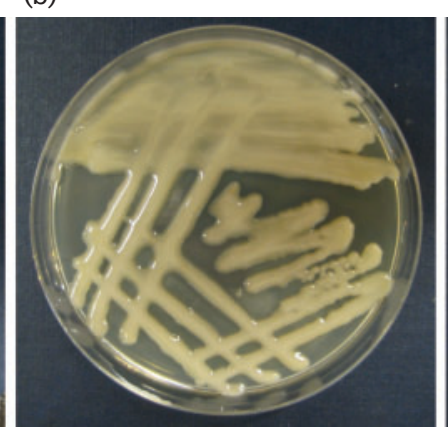

(c)

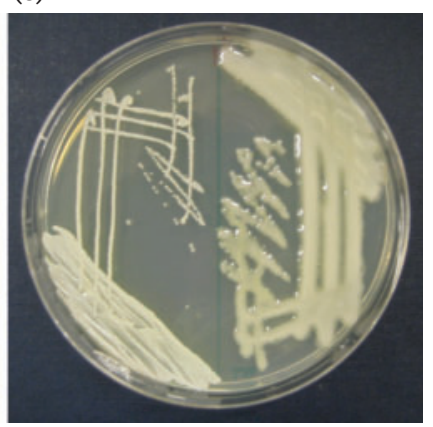

Fig. 1. Growth of $B$. ambifaria AMMD on (a) nutrient agar (non-mucoid); (b) onion agar (mucoid), and (c) comparison of $A M M D$ bce $B$ mutant (left) and AMMD wild-type (right) on mannitol agar.

\section{The onion factor}

Attempts were made to identify the 'onion factor' responsible for inducing EPS biosynthesis by use of standard biochemical methods for extracting and fractionating phytochemicals. The causative factor was retained on drying in vacuo, and remained in the aqueous phase after partition with ethyl acetate at $\mathrm{pH} 7.0$ and $\mathrm{pH}$ 2.0. When further physicochemical analyses discounted the role of proteins and lipids, attention was turned to the carbohydrate content of onion extract. After preparative paper electrophoresis in buffers at $\mathrm{pH} 2.0$ and 6.5, the only biological activity recovered from paper strips co-migrated with the standard glucose, indicating the absence of ionizable functional groups such as phosphate, acid or amine (data not shown). Analytical paper chromatography and HPAE-PAD identified the major carbohydrate components as sucrose, glucose, fructose and fructans (Fig. 2). The HPAE-PAD chromatograms in Fig. 2 show characteristic peaks of glucose, fructose and sucrose. Sucrose breaks down to fructose and glucose upon mild hydrolysis, as do the fructans to fructose. Fructose breaks down under complete hydrolysis as expected for a ketose sugar, whilst the aldose sugar glucose remains stable. The ability of these and related compounds to stimulate EPS biosynthesis in Bcc was then investigated (Table 1). Glycerol and mannitol were included as these sugar alcohols have previously been noted to enhance EPS biosynthesis in Pseudomonas aeruginosa (Whitchurch et al., 1996) and the Bcc (Sage et al., 1990; Zlosnik et al., 2008). Glucitol was included because of its close degradative relationship with fructose and mannitol (Allenza et al., 1982). These experiments showed that within a particular Bcc species, EPS biosynthesis was strain-specific and that the most potent inducers of EPS were fructose and all alditols tested, most significantly mannitol and glucitol, as well as the cyclitol myo-inositol. Importantly, the profile of EPS biosynthesis production with these sugars was similar to that observed with onion extract (Table 1). EPS biosynthesis was not observed on agar containing yeast extract alone, nor in the presence of glucose (Table 1), galactose, lactose or maltose (data not shown) with any Bcc strains tested.

\section{Investigation of the molecular basis for EPS biosynthesis}

With the exception of the single B. stabilis strain tested, all Bcc species were shown to be capable of producing EPS when grown on onion agar (Table 1 and Supplementary Tables S1-S3), suggesting the presence of a conserved EPS biosynthetic gene cluster. Therefore genome sequences representing five Bcc species (B. ambifaria, B. multivorans, B. vietnamiensis, B. dolosa and Burkholderia sp. 383) were examined to determine if two putative EPS gene clusters within B. cenocepacia J2315, the bce gene cluster (Moreira et al., 2003) and the wcb gene cluster (Parsons et al., 2003), are conserved across the Bcc. The $w c b$ gene cluster was found to be poorly conserved, with between one-third and one-half of J2315 ORFs having no direct homologues within the species examined (data not shown). Notably, within the EPS-producing B. ambifaria AMMD strain, over half of the J2315 $w c b$-associated ORFs have no homologues, and the remaining homologous ORFs are not organized within a gene cluster. In contrast, the bce gene cluster was conserved across all species examined, in terms of both sequence homology and organization of ORFs (Supplementary Fig. S1). In the course of these genome comparisons, a third putative polysaccharide biosynthetic gene cluster was observed on chromosome 2 of $B$. cenocepacia J2315. This cluster encodes two putative EPS transporter proteins (BCAM1330 and BCAM1331), an acyltransferase (BCAM1333), several glycosyltransferases (BCAM1335, BCAM1337, BCAM1338), a polysaccharide biosynthesis protein (BCAM1336) and a mannose-6phosphate isomerase (BCAM1340). This cluster is conserved amongst several Bcc species, albeit to a lesser extent than the bce gene cluster (data not shown). In the EPSproducing B. ambifaria AMMD, this gene cluster maps to ORFs Bamb_3621 through to Bamb_3629. 

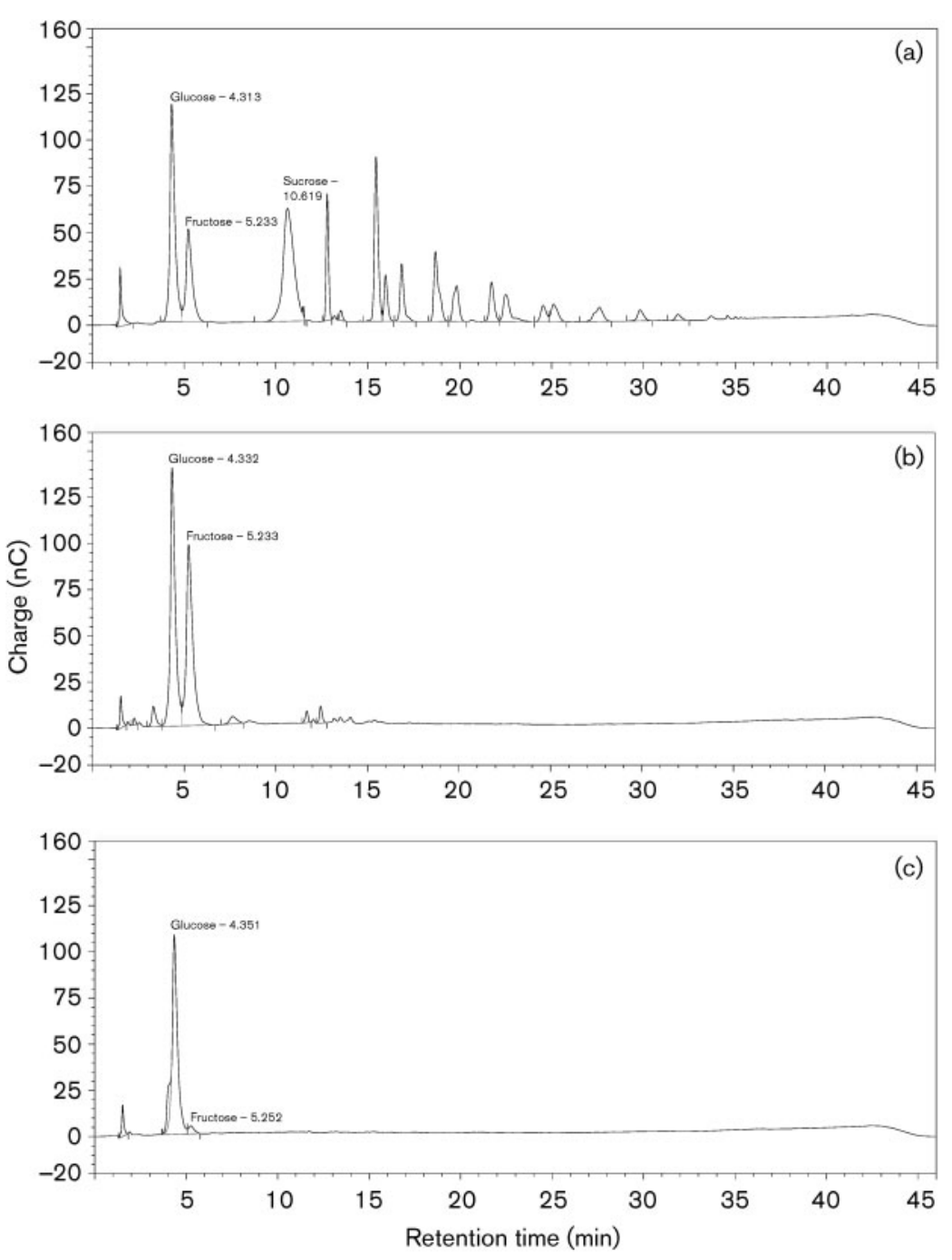

Fig. 2. HPAE-PAD chromatograms: Carbopac PA-100 column separation of sugars in onion extract. (a) Crude onion extract; (b) onion extract hydrolysed by $2 \mathrm{M}$ TFA $60^{\circ} \mathrm{C} 1 \mathrm{~h}$; (c) onion extract hydrolysed by $2 \mathrm{M}$ TFA $120^{\circ} \mathrm{C}$ $1 \mathrm{~h}$. Glucose, fructose and sucrose peaks were clearly identified based on standards (not shown), and hydrolysis pattern. Peaks with retention times between 12 and $36 \mathrm{~min}$ appear to be oligosaccharides of fructose based on their degradation to fructose under mild hydrolysis.
To investigate which polysaccharide gene cluster is induced by growth on mannitol, the expression of representative genes from two distinct EPS gene clusters was assessed in $B$. ambifaria AMMD grown in the presence and absence of mannitol. The genes studied each encode homologues of the Wza EPS export protein: Bamb_5549 of the bce gene cluster (equivalent to bceE, BCAM0858 of B. cenocepacia J2315), and Bamb_3621 of the novel putative polysaccharide gene cluster described above (BCAM1330 of $B$. cenocepacia J2315). As shown in Fig. 3, expression of Bamb_3621 was not observed under either growth condition. In contrast, expression of the bceE homologue (Bamb_5549) was clearly induced by the presence of mannitol. We therefore focused on the bce gene cluster to investigate why some Bcc isolates, most notably those of the $B$. cenocepacia ET12 lineage, failed to produce EPS under any growth conditions.

A PCR assay was designed to screen isolates for an $11 \mathrm{bp}$ deletion in the $b c e B$ gene that has been suggested to be responsible for loss of EPS production in the CF isolate $B$. cenocepacia J2315 (Moreira et al., 2003). Of the panel of strains shown in Table 1, only the B. cenocepacia ET12 isolates J2315, K56-2 and BC7 harbour the 11 bp deletion (data not shown). This result prompted us to test a panel of B. cenocepacia IIIA strains containing both ET12 and nonET12 isolates. There was a clear correlation between the $11 \mathrm{bp}$ deletion and the presence of both $c b l A$ (cable pilus) and BCESM (B. cepacia epidemic strain marker), indicating that this deletion is a conserved feature within the ET12 lineage (Fig. 4). In our study, with the exception of strain E3051, the presence of the deletion correlated with the lack of EPS production in all B. cenocepacia IIIA isolates examined (Fig. 4; Supplementary Table S1). Furthermore, the $11 \mathrm{bp}$ deletion in the $b c e B$ gene was not observed in any B. cenocepacia IIIB or B. multivorans isolates studied (see Supplementary Tables S2 and S3).

Using proven methods for the complementation of gene function in B. cenocepacia (Ortega et al., 2005), attempts were made to complement $\mathrm{BceB}$ function within $B$. cenocepacia K56-2 by introducing a wild-type bceB ORF 


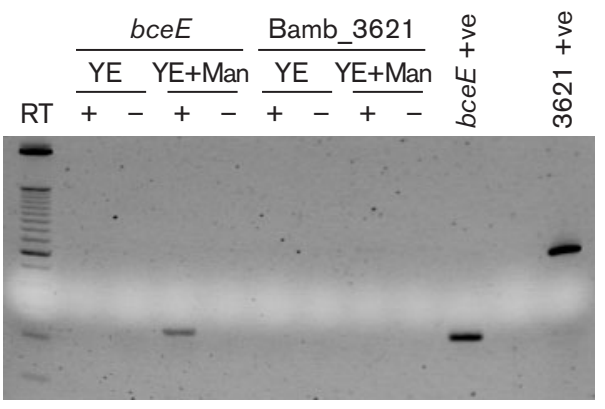

Fig. 3. Induction of the bce gene cluster by mannitol. RT-PCR analysis was performed to assess the expression of two distinct wza homologues (bceE and Bamb_3621) of B. ambifaria AMMD in the absence and presence of mannitol. The bce $E$ gene (Bamb_5549) is located within the bce gene cluster, whilst Bamb_3621 is located within a distinct putative EPS biosynthetic gene cluster. Expression was assessed in yeast extract (YE) and yeast extract supplemented with $2 \%(\mathrm{w} / \mathrm{v})$ mannitol (YE+Man). Expression of Bamb_3621 was not detected in YE or YE + Man. In contrast, bce $E$ expression was strongly induced by growth in $\mathrm{YE}+$ Man. For each sample, RT and non-RT reactions $(+/-)$ are shown alongside each other. Genomic DNA positive controls are shown for each gene (bceE +ve, $3621+\mathrm{ve})$.

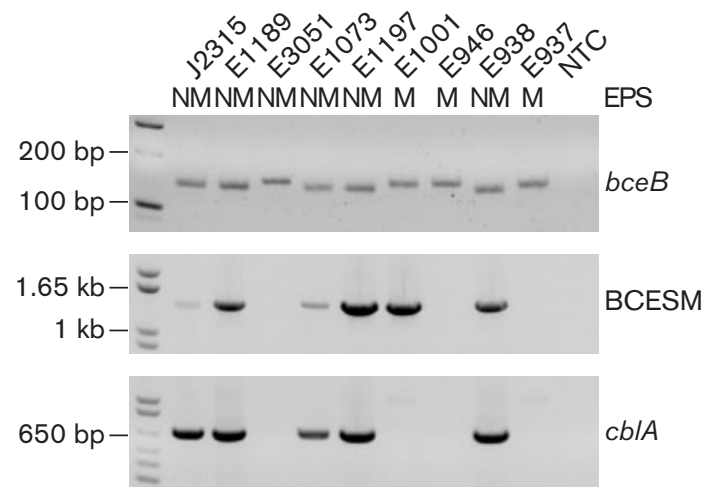

Fig. 4. bceB PCR analysis of representative $B$. cenocepacia IIIA isolates. For each isolate, EPS production is indicated as mucoid (M) or non-mucoid (NM) alongside PCR analysis of bceB, and the epidemic strain markers $c b / A$ and BCESM. All PCR products are pictured alongside the $1 \mathrm{~kb}$ Plus DNA Ladder (Invitrogen). NTC, no template control. The bceB PCR assay yields a 151 bp product from wild-type sequence and a $140 \mathrm{bp}$ product from sequence harbouring an $11 \mathrm{bp}$ deletion (as previously reported within J2315). With one exception (E3051), lack of EPS production within $B$. cenocepacia IIIA isolates correlated with the $11 \mathrm{bp}$ deletion within $b c e B$. This deletion was only observed within strains that were positive for both $c b / A$ and BCESM, indicating it to be a conserved feature of the ET12 lineage. The bce $B$ deletion was not observed in a strain harbouring just one of the two epidemic strain markers (E1001). amplified from B. cenocepacia PC184. Whilst expression of 6His-tagged BceB was detected in E. coli C41(DE3) cells, previously shown to support expression of membranebound proteins (Miroux \& Walker, 1996), we were unable to detect expression within B. cenocepacia K56-2 (data not shown). Consequently, we chose to disrupt the $b c e B$ ORF of an EPS-producer. Insertional inactivation of the $b c e B$ gene resulted in loss of EPS production in B. ambifaria AMMD when grown on mannitol agar (Fig. 1c) and on onion agar (data not shown).

\section{DISCUSSION}

In this study, we returned to the original Bcc host and report that Bcc isolates previously considered non-mucoid produce copious amounts of EPS when onion tissue is provided as the sole nutrient, highlighting the metabolic potential of this group of organisms. This novel and surprising observation is not species-specific, and is exhibited by isolates of both clinical and environmental origin. The onion components responsible for EPS induction are primarily the carbohydrates sucrose, fructose and fructans. Additionally, all alcohol sugars tested are able to induce EPS production, in particular mannitol and glucitol.

The reasons why EPS biosynthesis is readily induced by onion extracts and, in particular, by fructose and the hexitol sugars mannitol and glucitol require further investigation. An ability to respond to inulin was confined to certain strains, all of which responded well to fructose. This suggests that the fructan polysaccharide was only active in bacteria that can hydrolyse it to fructose. The ability of one strain (BTS7) to respond particularly strongly to inulin could possibly be due to its enhanced ability to hydrolyse inulin to fructose. Interestingly, the similar profile of EPS induction shown by fructose and alcohol sugars agrees well with a previous study which showed that the initial steps of utilization of these sugars in the Bcc differ from those in most other pseudomonads. Growth of $\mathrm{Bcc}$ on fructose involves active transport followed by fructokinase conversion to fructose 6-phosphate then degradation via the Entner-Doudoroff pathway (Allenza et al., 1982). Growth on mannitol and glucitol also requires this pathway following active uptake and intracellular oxidation to fructose.

The role of Bcc EPS as a putative virulence factor is unclear. The influence of mannitol and other alcohol sugars on EPS production suggests that the prevalence of EPS in the Bcc may have been underestimated. In addition, given the variety of human, animal, plant and other models studied, the role of EPS may be influenced by the host involved and the route of administration. EPS has been associated with altered Bcc clearance in a mouse model of infection (Conway et al., 2004), and EPS-deficient Bcc mutants displayed reduced mortality within a CGD mouse model (Sousa et al., 2007a). Similarly, a role for EPS in persistence 
in human airways is suggested by its capacity to scavenge reactive oxygen species and inhibit neutrophil chemotaxis (Bylund et al., 2006). In our study, EPS biosynthesis did not correlate with the ability to cause onion rot, which is perhaps to be expected since pectinases rather than EPS are likely to play the major role in the maceration of plant tissue. Cunha et al. (2004) did not observe a clear correlation between EPS biosynthesis in vitro and the ability of Bcc strains to establish chronic infections within the CF lung. Recent evidence, however, suggests a subtle and unexpected role for Bcc EPS in CF lung infection. Consistent with our findings, Zlosnik et al. (2008) reported that isolates of $B$. cenocepacia, the most virulent $\mathrm{Bcc}$ species, are most frequently non-mucoid. They also observed a mucoid to non-mucoid conversion in sequential isolates of Bcc from chronically infected CF patients. This apparent loss of mucoidy in vivo, and its absence in the virulent $B$. cenocepacia ET12 lineage, provides an intriguing contrast with the characteristic non-mucoid to mucoid conversion observed with alginate-producing $P$. aeruginosa. Zlosnik and colleagues suggest that Bcc EPS could be responsible for the persistence of $\mathrm{Bcc}$ in $\mathrm{CF}$ airways whilst loss of EPS leads to increased disease severity.

In our investigation of EPS biosynthesis determinants, we focused on the highly conserved bce gene cluster. Previously described by Moreira et al. (2003), the bce gene cluster has had several of its encoded proteins characterized (Ferreira et al., 2007; Sousa et al., 2007b; Videira et al., 2005) and is thought to be involved in cepacian biosynthesis. In the present study, we demonstrated induction of the bce gene cluster by mannitol, and found a clear correlation between the inability of representatives of the B. cenocepacia ET12 lineage to produce EPS and the presence of an $11 \mathrm{bp}$ deletion within the $b c e B$ gene, originally described within the genome sequence of $B$. cenocepacia J2315 (Moreira et al., 2003). Consistent with this correlation, insertional inactivation of $b c e B$, which encodes a glycosyltransferase, resulted in the loss of EPS production by $B$. ambifaria AMMD when grown on onion media. Combined, these observations highlight the pivotal role of the $b c e$ gene cluster in onion-induced EPS biosynthesis, and suggest that the observed EPS is cepacian. However, mutations elsewhere within the bce gene cluster, or in other EPS-related gene clusters, must be responsible for the lack of EPS biosynthesis in B. cenocepacia strain E3051 (Supplementary Table S1), and in other EPSnegative Bcc strains in our study which lack the $11 \mathrm{bp}$ deletion in bceB (Supplementary Tables S2 and S3).

The ability of hexoses and hexitols, in particular mannitol, to enhance EPS biosynthesis in the B. cepacia complex has disturbing implications for therapeutic intervention in CF. Recent attempts to improve airway clearance with hypertonic saline $5 \%(\mathrm{w} / \mathrm{v})$ have been handicapped by the problem of salty taste and the salt-sensitive nature of many antimicrobial peptides. Thus, attention has turned to the use of non-ionic osmolytes, including inhaled mannitol
(Daviskas et al., 2008; Robinson et al., 1999; Wills, 2007), which is marketed as Bronchitol (Pharmaxis). Robinson and colleagues acknowledged that the majority of $P$. aeruginosa and $\mathrm{Bcc}$ isolates are able to utilize mannitol as a carbon and energy source. However, they felt that the nutritional influence of mannitol as a therapeutic osmolyte would be minimal given the abundance of other nutrients already present in CF respiratory secretions. On a cautionary note, they state that this potential problem would need to be confirmed by quantitative microbiology. The potential induction of virulence determinants during osmolyte therapy has to our knowledge not been considered. Our results also provide justification for the continued exclusion of $\mathrm{CF}$ individuals known to be infected with Bcc from ongoing trials of inhaled mannitol (http://clinicaltrials.gov; identifier NCT00117208 and NCT00251056).

\section{ACKNOWLEDGEMENTS}

We thank Catherine Doherty (University of Edinburgh, UK) for the strain panels, Rosanna Hennessy (University of Edinburgh, UK) for the RT-PCR data, Paola Cescutti (Università di Trieste, Italy) for Burkholderia pyrrocinia BTS7 and B. cenocepacia BTS2, and Miguel Valvano (University of Western Ontario, Canada) for the suicide vector $\mathrm{pGP \Omega Tp}$ and complementation vector pSCRhaB3. S. J. B., A. R. B., J. R. W. G. and D. J. C. thank the CF Trust and the Big Lottery Fund, D. J. C. and D. J. C. also thank the Royal Society of Edinburgh, and S.C.F. and B.R.M. thank the Biotechnology and Biological Sciences Research Council for financial support.

\section{REFERENCES}

Allenza, P., Lee, Y. N. \& Lessie, T. G. (1982). Enzymes related to fructose utilization in Pseudomonas cepacia. J Bacteriol 150, 1348-1356.

Bernier, S. P., Silo-Suh, L., Woods, D. E., Ohman, D. E. \& Sokol, P. A. (2003). Comparative analysis of plant and animal models for characterization of Burkholderia cepacia virulence. Infect Immun 71, 5306-5313.

Burkholder, W. (1950). Sour skin, a bacterial rot of onion bulbs. Phytopathology 40, 115-117.

Bylund, J., Burgess, L. A., Cescutti, P., Ernst, R. K. \& Speert, D. P. (2006). Exopolysaccharides from Burkholderia cenocepacia inhibit neutrophil chemotaxis and scavenge reactive oxygen species. $J$ Biol Chem 281, 2526-2532.

Coenye, T., Vandamme, P., LiPuma, J. J., Govan, J. R. \& Mahenthiralingam, E. (2003). Updated version of the Burkholderia cepacia complex experimental strain panel. J Clin Microbiol 41, 2797-2798.

Conway, B. A., Chu, K. K., Bylund, J., Altman, E. \& Speert, D. P. (2004). Production of exopolysaccharide by Burkholderia cenocepacia results in altered cell-surface interactions and altered bacterial clearance in mice. J Infect Dis 190, 957-966.

Cunha, M. V., Sousa, S. A., Leitao, J. H., Moreira, L. M., Videira, P. A. \& Sa-Correia, I. (2004). Studies on the involvement of the exopolysaccharide produced by cystic fibrosis-associated isolates of the Burkholderia cepacia complex in biofilm formation and in persistence of respiratory infections. J Clin Microbiol 42, 3052-3058. 
Daviskas, E., Anderson, S. D., Eberl, S. \& Young, I. H. (2008). Effect of increasing doses of mannitol on mucus clearance in patients with bronchiectasis. Eur Respir J 31, 765-772.

Eshdat, Y. \& Mirelman, D. (1972). An improved method for the recovery of compounds from paper chromatograms. J Chromatogr 65, $458-459$.

Ferreira, A. S., Leitao, J. H., Sousa, S. A., Cosme, A. M., Sa-Correia, I. \& Moreira, L. M. (2007). Functional analysis of Burkholderia cepacia genes $b c e D$ and $b c e F$, encoding a phosphotyrosine phosphatase and a tyrosine autokinase, respectively: role in exopolysaccharide biosynthesis and biofilm formation. Appl Environ Microbiol 73, 524-534.

Flannagan, R. S., Aubert, D., Kooi, C., Sokol, P. A. \& Valvano, M. A. (2007). Burkholderia cenocepacia requires a periplasmic HtrA protease for growth under thermal and osmotic stress and for survival in vivo. Infect Immun 75, 1679-1689.

Fry, S. C. (2000). The Growing Plant Cell Wall: Chemical and Metabolic Analysis. Caldwell, NJ: The Blackburn Press.

Goldberg, J. B. (2007). Polysaccharides of Burkholderia spp. In Burkholderia: Molecular Microbiology and Genomics, pp. 93-110. Edited by T. Coenye \& P. Vandamme. Norwich, UK: Horizon Bioscience.

Govan, J. R. W. (2006). Burkholderia cepacia complex and Stenotrophomonas maltophilia. In Cystic Fibrosis in the 21st Century: Progress in Respiratory Research, pp. 145-152. Edited by A. Bush, E. W. F. W. Alton, J. C. Davies, U. Griesenbach \& A. Jaffe. Basel: Karger.

Govan, J. R. \& Deretic, V. (1996). Microbial pathogenesis in cystic fibrosis: mucoid Pseudomonas aeruginosa and Burkholderia cepacia. Microbiol Rev 60, 539-574.

Govan, J. R., Brown, P. H., Maddison, J., Doherty, C. J., Nelson, J. W., Dodd, M., Greening, A. P. \& Webb, A. K. (1993). Evidence for transmission of Pseudomonas cepacia by social contact in cystic fibrosis. Lancet 342, 15-19.

Herasimenka, Y., Benincasa, M., Mattiuzzo, M., Cescutti, P., Gennaro, R. \& Rizzo, R. (2005). Interaction of antimicrobial peptides with bacterial polysaccharides from lung pathogens. Peptides 26, 1127-1132.

Mahenthiralingam, E., Simpson, D. A. \& Speert, D. P. (1997), Identification and characterization of a novel DNA marker associated with epidemic Burkholderia cepacia strains recovered from patients with cystic fibrosis. J Clin Microbiol 35, 808-816.

Mahenthiralingam, E., Coenye, T., Chung, J. W., Speert, D. P., Govan, J. R., Taylor, P. \& Vandamme, P. (2000). Diagnostically and experimentally useful panel of strains from the Burkholderia cepacia complex. J Clin Microbiol 38, 910-913.

Mahenthiralingam, E., Urban, T. A. \& Goldberg, J. B. (2005). The multifarious, multireplicon Burkholderia cepacia complex. Nat Rev Microbiol 3, 144-156.

Miroux, B. \& Walker, J. E. (1996). Over-production of proteins in Escherichia coli: mutant hosts that allow synthesis of some membrane proteins and globular proteins at high levels. J Mol Biol 260, 289-298.

Moreira, L. M., Videira, P. A., Sousa, S. A., Leitao, J. H., Cunha, M. V. \& Sa-Correia, I. (2003). Identification and physical organization of the gene cluster involved in the biosynthesis of Burkholderia cepacia complex exopolysaccharide. Biochem Biophys Res Commun 312, 323-333.
Ortega, X., Hunt, T. A., Loutet, S., Vinion-Dubiel, A. D., Datta, A., Choudhury, B., Goldberg, J. B., Carlson, R. \& Valvano, M. A. (2005). Reconstitution of O-specific lipopolysaccharide expression in Burkholderia cenocepacia strain J2315, which is associated with transmissible infections in patients with cystic fibrosis. J Bacteriol 187, 1324-1333.

Parsons, Y. N., Banasko, R., Detsika, M. G., Duangsonk, K., Rainbow, L., Hart, C. A. \& Winstanley, C. (2003). Suppressionsubtractive hybridisation reveals variations in gene distribution amongst the Burkholderia cepacia complex, including the presence in some strains of a genomic island containing putative polysaccharide production genes. Arch Microbiol 179, 214-223.

Robinson, M., Daviskas, E., Eberl, S., Baker, J., Chan, H. K., Anderson, S. D. \& Bye, P. T. (1999). The effect of inhaled mannitol on bronchial mucus clearance in cystic fibrosis patients: a pilot study. Eur Respir J 14, 678-685.

Sage, A., Linker, A., Evans, L. R. \& Lessie, T. G. (1990). Hexose phosphate metabolism and exopolysaccharide formation in Pseudomonas cepacia. Curr Microbiol 20, 191-198.

Sajjan, U. S., Sun, L., Goldstein, R. \& Forstner, J. F. (1995). Cable $(c b l)$ type II pili of cystic fibrosis-associated Burkholderia (Pseudomonas) cepacia: nucleotide sequence of the cblA major subunit pilin gene and novel morphology of the assembled appendage fibers. $J$ Bacteriol 177, 1030-1038.

Sist, P., Cescutti, P., Skerlavaj, S., Urbani, R., Leitao, J. H., SaCorreia, I. \& Rizzo, R. (2003). Macromolecular and solution properties of cepacian: the exopolysaccharide produced by a strain of Burkholderia cepacia isolated from a cystic fibrosis patient. Carbohydr Res 338, 1861-1867.

Sousa, S. A., Ulrich, M., Bragonzi, A., Burke, M., Worlitzsch, D., Leitão, J. H., Meisner, C., Eberl, L., Sá-Correia, I. \& Döring, G. (2007a). Virulence of Burkholderia cepacia complex strains in gp91phox $^{-1-}$ mice. Cell Microbiol 9, 2817-2825.

Sousa, S. A., Moreira, L. M., Wopperer, J., Eberl, L., Sá-Correira, I. \& Leitão, J. H. (2007b). The Burkholderia cepacia bceA gene encodes a protein with phosphomannose isomerase and GDP-D-mannose pyrophosphorylase activities. Biochem Biophys Res Commun 353, 200-206.

Videira, P. A., Garcia, A. P. \& Sa-Correia, I. (2005). Functional and topological analysis of the Burkholderia cenocepacia priming glucosyltransferase BceB, involved in the biosynthesis of the cepacian exopolysaccharide. J Bacteriol 187, 5013-5018.

Whitchurch, C. B., Alm, R. A. \& Mattick, J. S. (1996). The alginate regulator $\mathrm{AlgR}$ and an associated sensor FimS are required for twitching motility in Pseudomonas aeruginosa. Proc Natl Acad Sci U S A 93, 9839-9843.

Wills, P. J. (2007). Inhaled mannitol in cystic fibrosis. Expert Opin Investig Drugs 16, 1121-1126.

Zlosnik, J. E., Hird, T. J., Fraenkel, M. C., Moreira, L. M., Henry, D. A. \& Speert, D. P. (2008). Differential mucoid exopolysaccharide production by members of the Burkholderia cepacia complex. J Clin Microbiol 46, 1470-1473.

Edited by: P. Cornelis 